\title{
Treatment Patterns of New Users of Fluticasone Furoate/Vilanterol in Asthma and COPD in UK Primary Care: Retrospective Cohort Study
}

\author{
Daniel Dedman (D) - Sonia J. Coton - Rebecca E. Ghosh • \\ Wilhelmine Meeraus • Courtney Crim • Catherine Harvey • \\ Justyna Amelio · Sarah H. Landis
}

Received: January 24, 2019 / Published online: April 24, 2019

(C) The Author(s) 2019

\section{ABSTRACT}

Introduction: This retrospective database study explored treatment patterns and potential offlabel prescribing among patients newly prescribed fluticasone furoate/vilanterol (FF/VI) in a UK primary care setting.

Enhanced digital features To view enhanced digital features for this article go to https://doi.org/10.6084/ m9.figshare.7964555.

Electronic supplementary material The online version of this article (https://doi.org/10.1007/s41030019-0092-z) contains supplementary material, which is available to authorized users.

D. Dedman $(\bowtie) \cdot$ S. J. Coton · R. E. Ghosh Clinical Practice Research Datalink (CPRD), Medicines and Healthcare Products Regulatory Agency (MHRA), London, UK

e-mail: Daniel.Dedman@mhra.gov.uk

W. Meeraus · S. H. Landis

GlaxoSmithKline plc, Epidemiology, Value Evidence and Outcomes, Stockley Park, Uxbridge, UK

C. Crim

GlaxoSmithKline plc, Research and Development, Research Triangle Park, NC, USA

C. Harvey

GlaxoSmithKline plc, Global Clinical Safety and Pharmacovigilance, Stockley Park, Uxbridge, UK

J. Amelio

GlaxoSmithKline plc, Epidemiology, Value Evidence and Outcomes, Stevenage, UK
Methods: In Europe, FF/VI is approved in two strengths: $100 / 25 \mu \mathrm{g}$ for adults with chronic obstructive pulmonary disease (COPD) and $100 / 25 \mu \mathrm{g}$ or $200 / 25 \mu \mathrm{g}$ for treatment of asthma in patients aged 12 or older. Using electronic health records from the Clinical Practice Research Datalink, new users of FF/VI or other inhaled corticosteroid/long-acting beta-agonist fixed-dose combination products were identified and classified into one of three groups: COPD diagnosis, asthma diagnosis, and other diagnosis (not COPD or asthma).

Results: During 2014-2015, 4373 patients initiated FF/VI: 3380 on FF/VI 100/25 (65\% in the COPD diagnosis group) and 993 on FF/VI 200/25 (51\% in the asthma diagnosis group). During up to 12 months of follow-up, the median number (interquartile range) of prescriptions of the index strength issued per patient was 7 (2-8) for FF/VI 100/25 and 5 (2-8) for FF/VI 200/25; most new users did not change from the index strength prescribed (93.0\% COPD; $89.7 \%$ asthma, of all patients initiating treatment with FF/VI). Potential offlabel $\mathrm{FF} / \mathrm{VI}$ prescribing in children $<12$ years old was rare $(<0.29 \%$ in the combined asthma and other diagnosis groups), and up to one in five new users of FF/VI with COPD were potentially prescribed FF/VI 200/25 off-label during the study period. Much of the potential off-label prescribing in COPD occurred in patients with a history of asthma, those 
presenting with greater disease severity, and/or prior treatment with high-dose steroids.

Conclusions: The prescription of FF/VI is rare in children under 12 years of age in the UK, according to our findings, but up to one in five COPD patients in the UK may have been prescribed FF/VI 200/25, some of which may have been off-label.

Funding: This study was funded by GlaxoSmithKline plc (study 205052).

Study Registration: GlaxoSmithKline plc Clinical Trial Registry study number 205052.

Keywords: Asthma; Chronic obstructive pulmonary disease (COPD); Fluticasone furoate/vilanterol (FF/VI); Primary care

\section{INTRODUCTION}

Inhaled corticosteroids (ICS) and long-acting beta-agonists (LABAs) are mainstays of treatment for chronic obstructive pulmonary disease (COPD) and asthma, both of which are common respiratory diseases globally $[1,2]$. There is strong evidence that combining an ICS with a LABA is more effective at reducing exacerbations and improving lung function and health status in patients with stable COPD than each component individually [3-7]. In asthma, the addition of a LABA to an ICS improves lung function and symptom scores while reducing the number of asthma exacerbations and nocturnal asthma symptoms [8], and is thus preferred to increasing the ICS dose to achieve asthma control [2].

Fluticasone furoate/vilanterol (FF/VI) is a fixed-dose combination (FDC) ICS/LABA for once-daily inhalation that is available in two dose strengths, $100 / 25 \mu \mathrm{g}$ and $200 / 25 \mu \mathrm{g}$. In Europe, the $100 / 25 \mu \mathrm{g}$ strength is indicated for the symptomatic treatment of adults with COPD who have a history of exacerbations despite bronchodilator therapy. Both strengths $(100 / 25 \mu \mathrm{g}$ and $200 / 25 \mu \mathrm{g})$ are approved for regular treatment of patients with asthma aged $\geq 12$ years whose symptoms are not controlled with an ICS plus an inhaled short-acting $\beta_{2}$ agonist, or whose symptoms are adequately controlled with an ICS/LABA [9].
It is important to study how newly marketed drugs are prescribed in clinical practice following their launch, especially regarding possible off-label prescribing. While prescribers may be familiar with the class of medication, they may lack knowledge as to the correct use of the newly marketed product and assume it has a similar indication to other medications in the class, or they may extrapolate clinical trial data to populations that were not investigated. In the United Kingdom (UK), prescribed medication is commonly accessed via general practitioners (GPs) and is recorded in primary care electronic health records (EHR) maintained in general practice. These records also include interactions with secondary care. This observational study using EHR from the Clinical Practice Research Datalink (CPRD) was conducted to investigate treatment patterns among new users of FF/VI in the UK primary care setting, including potential indication for use and prescribing of concomitant medication. Two aspects of potential off-label prescribing were explored: FF/VI (any strength) in children aged $<12$ years, and FF/VI 200/25 in patients with evidence of a COPD diagnosis.

\section{METHODS}

\section{Study Design}

This retrospective, longitudinal, observational study utilised primary care EHR from CPRD, which holds data that are broadly representative in terms of age, sex, and ethnicity of the UK population [10]. FF/VI was first marketed in the UK in January 2014. The study population included new users of FF/VI or another ICS/ LABA FDC (fluticasone propionate/salmeterol xinafoate, budesonide/formoterol, beclometasone/formoterol, and fluticasone propionate/formoterol) during the 2-year inclusion period of 1 January 2014 to 31 December 2015 (the date of the first prescription was the index date). New prescribing was defined for a specific FDC product as never having had a prescription for $\mathrm{FF} / \mathrm{VI}$ or another index ICS/LABA FDC recorded previously (prior prescribing of a different ICS/LABA FDC and concomitant 
prescribing of other respiratory medications on the index date were permitted). A patient could contribute information on more than one index medication if they met the "new user" definition for multiple medications during the inclusion period. Patients were required to have at least 12 months of data prior to the index date to allow the characterisation of disease status, demographics, and clinical characteristics. Patients were followed from their index date until the first of the following events: (1) 12 months post index date, (2) death (censored), or (3) leaving their GP practice (censored), potentially allowing up to 12 months of follow-up time. The other ICS/LABA FDC cohort provided a benchmark for patient characteristics of new users of FF/VI, but was not included as a formal comparison group (no statistical comparisons were made).

New users were classified sequentially into three mutually exclusive diagnosis groups using validated coding lists and algorithms [11, 12]. First, a COPD diagnosis group was defined as new users with a COPD diagnosis recorded any time up to the end of their follow-up and aged $\geq 35$ years at the time of their first ever recorded COPD diagnosis. Patients in the COPD diagnosis group were further stratified by 'asthma history' (i.e. an asthma diagnosis recorded prior to and including the index date). Next, the asthma diagnosis group was defined as those patients who did not meet the criteria for COPD and had an asthma diagnosis recorded at any time up to the end of their follow-up. The other diagnosis group was defined as patients who did not meet the criteria for either the COPD or asthma groups.

\section{Demographics of New Users of FF/VI or Another ICS/LABA FDC}

Patient characteristics (gender, age, smoking status, and body mass index) at the index date and the prescribing of other asthma or COPD medications in the 12 months prior to the index date were described; for the ICS category, the highest dose prescribed (high/medium/low) was recorded based on Global Initiative for Asthma conversion guidelines [13]. Disease burden variables for the COPD group were described in the 12 months prior to the index date (unless otherwise noted): (1) forced expiratory volume in the first second $\left(\mathrm{FEV}_{1}\right)$ as a percentage of that predicted, and $\mathrm{FEV}_{1} /$ forced vital capacity (FVC) ratio based on the most recent lung function test in the 24 months prior to the index date (note: the primary care record does not always record pre- or postbronchodilator status); (2) Medical Research Council (MRC) dyspnoea score; and (3) acute exacerbations of COPD using a validated algorithm [14]. For the primary analysis of acute exacerbations of COPD, we captured episodes recorded in the GP database alone; a sensitivity analysis of a subset of patients whose GP-recorded data could be linked to the Hospital Episode Statistics (HES) was conducted to more fully capture exacerbation episodes that required hospitalisation (see the Electronic supplementary material, ESM, for more detail). Exacerbation episodes were defined as COPD-specific treatments with antibiotics combined with oral corticosteroid (OCS) and/or medical diagnosis codes for COPD exacerbations or acute bronchitis, or as a hospitalisation for COPD. For the asthma diagnosis group, asthma exacerbations in the 12 months prior to the index date were defined as a prescription for an OCS within \pm 14 days of an asthma code or an asthma hospital referral or asthma exacerbation events requiring hospitalisation. Asthma exacerbation episodes were reported in the main analysis using only the GP-recorded data, and in the sensitivity analysis for patients whose data could be linked to HES data.

\section{FF/VI Prescribing Patterns}

For new users of FF/VI 100/25 and FF/VI 200/25, the total count and median [interquartile range (IQR)] number of FF/VI prescriptions over up to 12 months of follow-up was calculated. Among patients who received at least two prescriptions of FF/VI (any strength), the proportion of new users with an escalation or reduction in FF/VI strength during the follow-up was reported by diagnosis group. Concomitant prescribing of other respiratory therapies at the index date was also identified, with concomitant therapy defined as at least two continuous prescriptions for the other respiratory therapy that started either before or up to 30 days after the index 
date and overlapped for at least 30 days with the index FF/VI treatment.

\section{Potential Off-Label Prescribing of FF/VI}

Potential off-label prescribing of FF/VI (any strength) in children aged $<12$ years was calculated as the number of patients aged $<12$ years old who received FF/VI (any strength) divided by all patients in the asthma and other diagnosis groups (by definition, all patients in the COPD diagnosis group were aged $\geq 35$ years and therefore excluded from the denominator population).

Potential off-label prescribing of FF/VI 200/25 in patients with evidence of COPD was considered using two alternative approaches that accounted for COPD patients with and without an 'asthma history', as it is not possible to determine from a retrospective database whether FF/VI 200/25 was prescribed to treat COPD or asthma. The first approach provides upper bound estimates, assuming that all COPD patients with an asthma history receive FF/VI 200/25 for treatment of their COPD either (1) at the index date (number of patients with COPD prescribed FF/VI 200/25 as their index prescription divided by all patients in the COPD diagnosis group), or (2) at any time during follow-up (number of patients with COPD prescribed FF/ VI 200/25 at any time during follow-up divided by all patients in the COPD diagnosis group). The second approach provides lower-bound estimates by assuming that COPD patients with an asthma history are prescribed FF/VI 200/25 for treatment of their asthma. For this approach, the proportion was calculated: (1) at the index date (number of patients with COPD and no asthma history prescribed FF/VI 200/25 as their index prescription divided by all patients in the COPD diagnosis group), or (2) at any time during follow-up (number of patients with COPD without asthma history prescribed FF/VI 200/25 at any time during follow-up divided by all patients in the COPD diagnosis group).

\section{Statistical Analysis}

Patient characteristics were described using mean (standard deviation) and median (IQR) for quantitative variables and numbers $(n)$, and proportions (\%) for categorical variables. COPD and asthma exacerbations during the 12 months prior to the index date were described as $0,1,2+$ events and were expressed as rates per person-year with the corresponding 95\% confidence interval (CI). When data were missing, the number of patients with missing data was reported, but these patients were excluded from the summary statistics.

\section{Ethics}

The CPRD contains only de-identified patient data. The CPRD has broad Health Research Authority National Research Ethics Service Committee ethics and governance approval for purely observational research using the primary care data and established data linkages. The study protocol (16_229R) was approved by the CPRD Independent Scientific Advisory Committee. Tables were adapted to suppress patient counts of less than five, in compliance with CPRD policy on managing anonymisation and the risk of identification in observational research.

\section{RESULTS}

New users of FF/VI were classified into one of three groups: COPD diagnosis (further stratified by asthma history), asthma diagnosis, and other diagnosis (not COPD or asthma). During the study period, 4373 patients initiated FF/VI: 3380 on FF/VI 100/25 (29\% COPD with an asthma history, 36\% COPD without an asthma history, 31\% asthma, 4\% other) and 993 on FF/ VI 200/25 (25\% COPD with an asthma history, 20\% COPD without an asthma history, 51\% asthma, $4 \%$ other) (Fig. 1, Table 1). During the same time period, 48,444 patients initiated other ICS/LABA FDCs, with just over half categorised as asthma patients (14\% COPD with an asthma history, 18\% COPD without an asthma history, $57 \%$ asthma, $11 \%$ other). The most common diagnostic codes in the other diagnosis group were related to upper or lower respiratory tract infections. Following initiation, about three-quarters of the patients contributed 


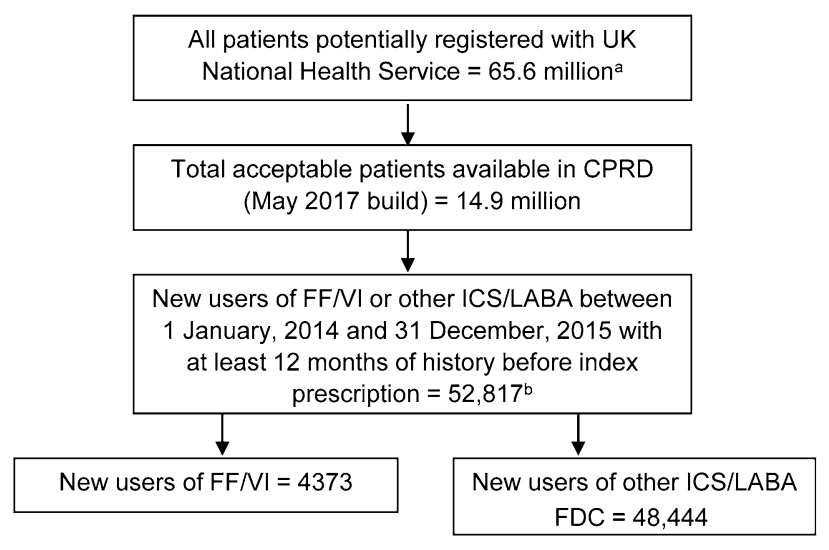

Fig. 1 CONSORT diagram. Superscript letter a indicates the mid-year estimate of the UK population for 2016 from the Office for National Statistics. Superscript letter $b$ indicates that patients contributed information on more than one treatment if they met the "new user" definition

data for the full 12-month follow-up period; a patient leaving their GP practice was the most common reason for censoring.

\section{Demographic Characteristics of New Users of FF/VI or Another ICS/LABA FDC}

Demographic characteristics are presented separately for the COPD, asthma, and other diagnosis groups (Table 1, Table S1 in the ESM). The mean age of new users of FF/VI or another ICS/ LABA FDC in the COPD diagnosis group was 68.1-69.4 years, and was marginally higher in the COPD without an asthma history diagnosis group (Table S1 in the ESM). The mean age of the asthma diagnosis group showed more variation over the three exposure cohorts (i.e. FF/VI 100/25, FF/VI 200/25, and other ICS/LABA FDC), ranging from 47.0 years for other ICS/ LABA FDC to 53.1 years for FF/VI 200/25.

Females comprised about half of new users in the COPD diagnosis group over the three exposure cohorts (range 46.7-50.4\%), while the proportion of females was greater in the asthma group (range $61.2 \%-66.5 \%$ ). The proportion of current smokers was highest in the COPD diagnosis group without an asthma history (range 39.9-43.4\%), followed by COPD patients with an asthma history (range 32.8-35.7\%), and was lowest among patients in the asthma for more than one medication during the inclusion period. CPRD Clinical Practice Research Datalink, FDC fixeddose combination, $F F / V I$ fluticasone furoate/vilanterol, $I C S / L A B A$ inhaled corticosteroid/long-acting beta agonist

diagnosis group (range 17.8-24.3\% (Table 1, Table S1 in the ESM).

\section{Prior and Concomitant Respiratory Medication Prescribing Among New Users of FF/VI or Another ICS/LABA FDC}

In the 12 months prior to the index date, FF/VI users were more likely to have received a different ICS/LABA medication $(69.1 \%$ for $\mathrm{FF} / \mathrm{VI}$ $100 / 25$ and $67.9 \%$ for FF/VI 200/25) than patients initiating another ICS/LABA FDC (42.0\%); past prescribing of long-acting muscarinic antagonist (LAMA) monotherapy and LABA/LAMA dual therapy was also greater for FF/VI users (Fig. S1 in the ESM). A large proportion of the COPD patients had been prescribed a high-dose ICS (alone or in combination with LABA) in the 12 months prior to initiating FF/VI 200/25 (37.9\% in COPD patients without an asthma history' and $57.2 \%$ in COPD patients with an asthma history). These proportions were similar in patients initiating FF/VI 100/25. Chronic use of OCS $(\geq 4$ prescriptions, with a maximum gap between two prescriptions equal to 30 days) was present in fewer than $10 \%$ of patients for both FF/VI strengths. Among patients in the asthma diagnosis group, nearly all were prescribed a shortacting bronchodilator (SABD) in the 12 months 


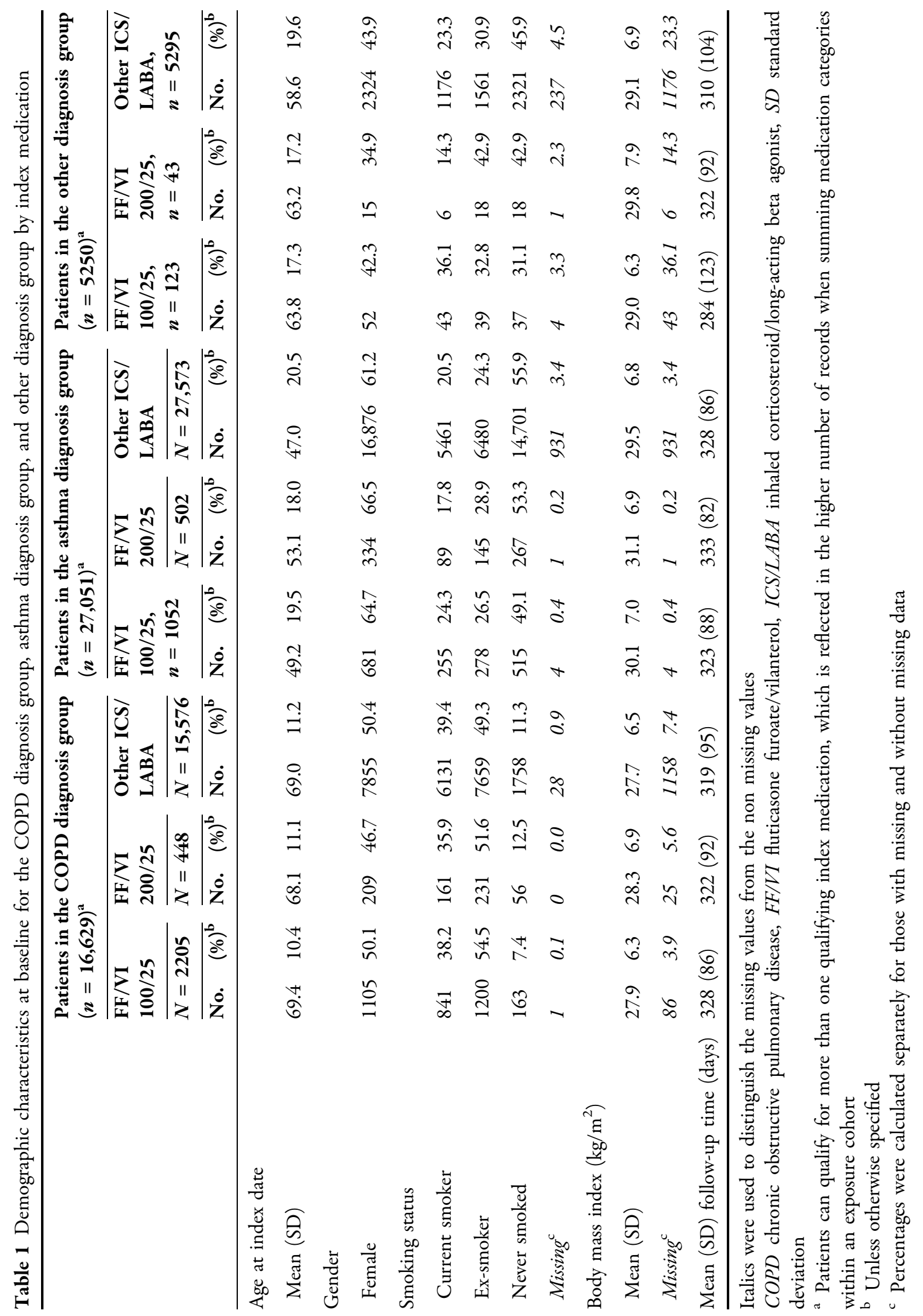




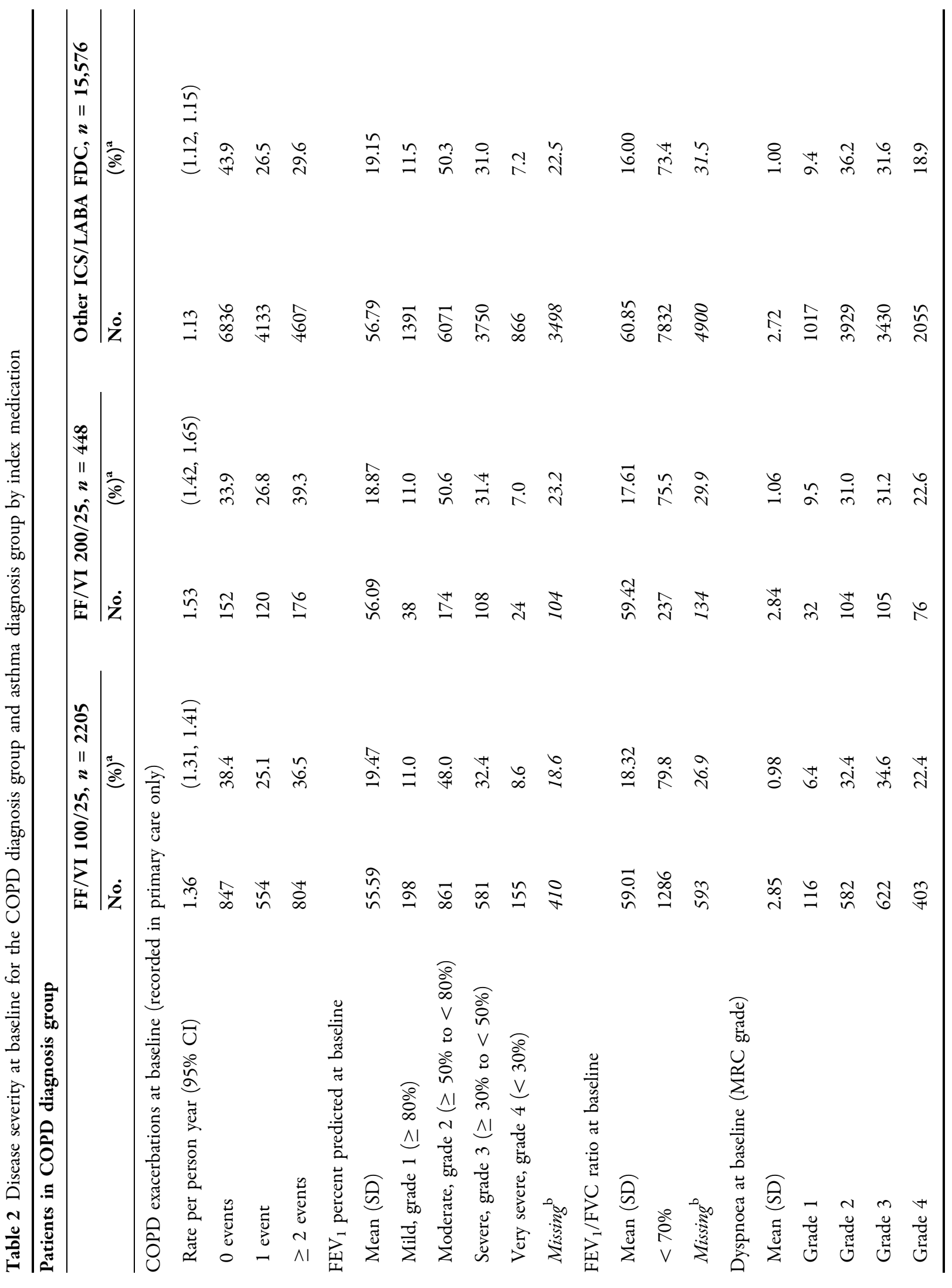




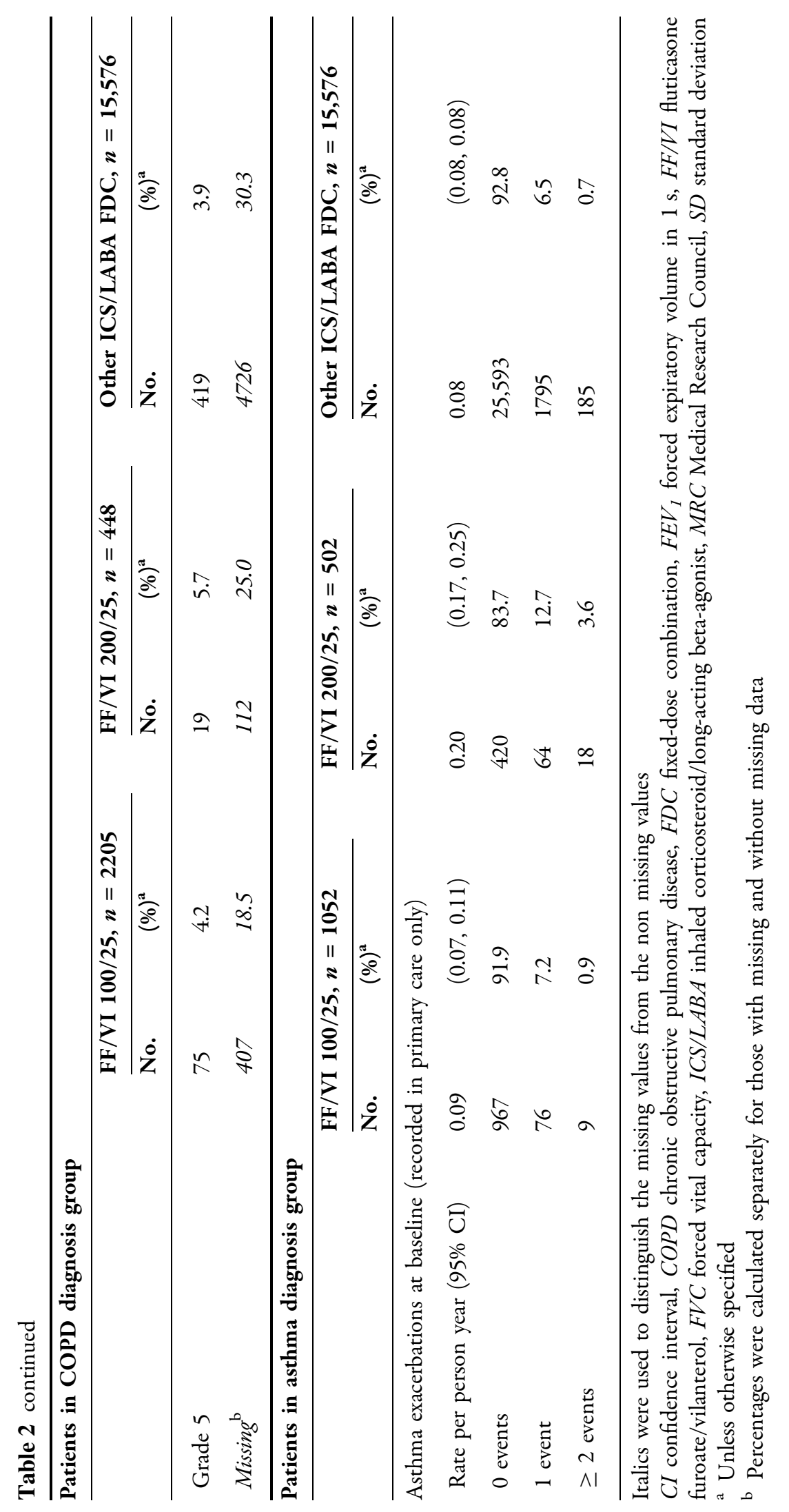




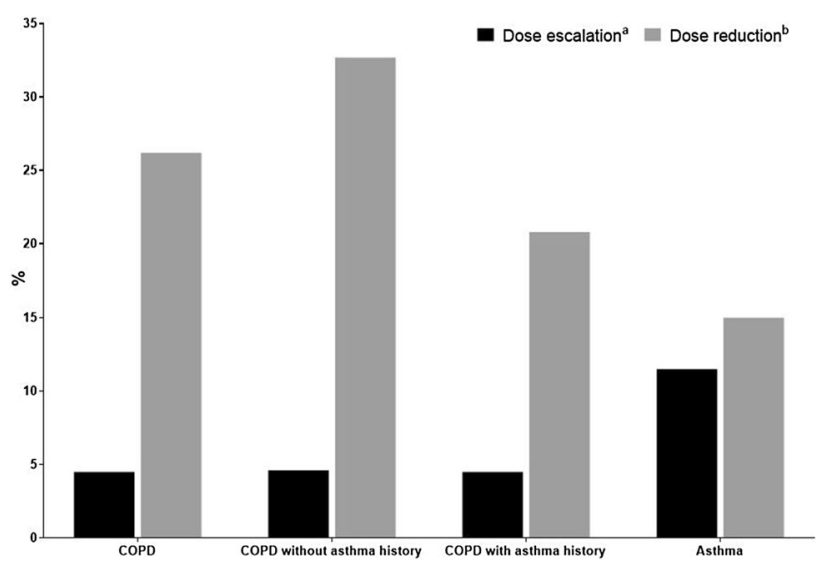

Fig. 2 Dose adjustment according to diagnosis group (among patients who received $\geq 2$ prescriptions of FF/ VI). Superscript letter $a$ indicates that the denominator includes new users of FF/VI 100/25 with at least two prescriptions of FF/VI (COPD $=1920$, COPD without an asthma history $=1050$, COPD with an asthma history $=870$, asthma $=853$ ). Superscript letter $b$ indicates

prior to the index date, and this was similar for FF/VI (any strength) and another LABA/ICS FDC. Past prescribing of another ICS/LABA was more common for the FF/VI group, especially the group initiating FF/VI 200/25, than for the LABA/ICS FDC group, whereas ICS monotherapy prescribing was highest for the LABA/ICS FDC group (Fig. S2 in the ESM).

Among patients in the COPD diagnosis group, LAMA was the most commonly prescribed concomitant maintenance medication (i.e. multiple inhaler triple therapy) at the index date $(59.7 \%$ for FF/VI $100 / 25$ and $51.8 \%$ for FF/ VI 200/25); concomitant LAMA prescribing was less frequent in the LABA/ICS FDC group $(45.4 \%)$. In the asthma diagnosis group, SABD concomitant prescribing was also the most common $(63.3 \%$ for FF/VI 100 and $58.6 \%$ for FF/VI 200), followed by leukotriene receptor antagonists (17.9\% for FF/VI 200/25; 9.5\% for FF/VI 100/25; 8.6\% for other LABA/ICS FDC).

\section{COPD and Asthma Disease Severity Among New Users of FF/VI or Another ICS/LABA FDC}

Among patients in the COPD group, new users of FF/VI 200/25 had the highest rates of COPD that the denominator includes new users of FF/VI 200/25 with at least two prescriptions of FF/VI (COPD $=378$, COPD without an asthma history $=171$, COPD with an asthma history $=207$, asthma $=414)$. COPD chronic obstructive pulmonary disease, $F F / V I$ fluticasone furoate/ vilanterol

exacerbation in the year prior to index date (1.53 per person-year, 95\% CI 1.42, 1.65) compared with new users of FF/VI 100/25 (1.36 per person-year, 95\% CI 1.31, 1.41) and new users of another ICS/LABA FDC (1.13 per person-year, 95\% CI 1.12, 1.15; Table 2). The HES-linked subsample, which facilitated the more complete capture of exacerbation episodes treated in secondary care, had slightly higher rates but followed the same pattern as the full CPRD sample (Table S2 in the ESM). Among this subset of patients, the rate of moderate COPD exacerbations was 1.53 per person per year $(95 \% \mathrm{CI}$ : $1.37,1.72$ ) for new users of FF/VI 200/25, 1.37 per person per year $(95 \%$ CI: $1.30,1.45)$ for new users of FF/VI 100/25, and 1.18 per person per year $(95 \%$ CI: $1.15,1.20)$ for new users of another ICS/LABA FDC. Airflow limitation as measured by $\mathrm{FEV}_{1}$ percent predicted was similar among the three drug exposure cohorts (Table 2), with $7-8 \%$ of patients with very severe, grade $4\left(\mathrm{FEV}_{1}<30 \%\right)$ airflow limitation. Breathlessness as measured by MRC dyspnoea score was more severe in COPD patients initiating FF/VI (100/25 or $200 / 25)$ than in COPD patients initiating another ICS/LABA FDC.

Rates of exacerbations in the asthma diagnosis group in the year prior to index date were 
also highest in new users of FF/VI 200/25 (0.20 per person-year, 95\% CI 0.17, 0.25) compared with FF/VI 100/25 (0.09 per person-year, 95\% CI $0.07,0.11)$, and ICS/LABA FDC (0.08 per personyear, 95\% CI 0.08, 0.08; Table 2). In the HESlinked subsample, the exacerbation rates were slightly higher but followed the same pattern as for the full CPRD sample (Table S2 in the ESM).

\section{Exposure to FF/VI or Another LABA/ICS FDC}

The median number of prescriptions of the index strength issued per patient during up to 12 months of follow-up was 7 (IQR 2-8) for FF/ VI 100/25 and 5 (IQR 2-8) for FF/VI 200/25. Between a fifth and a quarter of new users had only one recorded prescription of their index FF/VI strength during the study. Adherence to treatment was assessed for patients with the full 12 months of follow-up data, corresponding to approximately two-thirds of the COPD patients and one-third of the asthma patients (Table S3 in the ESM). In COPD, $62.0 \%$ and $46.8 \%$ of patients had a medication possession ratio and proportion of days covered by prescribed treatment, respectively, of at least $80 \%$. In asthma, $50.9 \%$ and $37.7 \%$ of patients had a medication possession ratio and proportion of days covered by prescribed treatment, respectively, of at least $80 \%$.

Most new users did not change from the index strength prescribed over the subsequent 12 months (93.0\% COPD; $89.7 \%$ asthma, of all patients initiating treatment with $\mathrm{FF} / \mathrm{VI}$ ). Among COPD patients, those initiating on FF/ VI 200/25 were more likely to receive just one prescription of this strength, compared with those initiating on FF/VI 100/25 (26.1\% vs. $13.7 \%$, respectively). For asthma patients, the proportion of FF/VI 100/25 and FF/VI 200/25 initiators with just one prescription of the index strength was similar $(22.6 \%$ and $21.5 \%$, respectively).

Amongst new users of FF/VI who had at least two prescriptions and for which it was possible to observe a change in strength, changes from FF/VI 200/25 to FF/VI 100/25 (i.e., a reduction in strength) were more frequent in the COPD diagnosis group than an escalation from FF/VI $100 / 25$ to the higher strength $(26.2 \%$ and $4.5 \%$, respectively), while in the asthma diagnosis group, reductions and escalations were equally likely (11.5\% vs. $15.0 \%$, respectively) (Fig. 2).

\section{Potential Off-Label Prescribing of FF/VI}

Fewer than five children aged $<12$ years received a prescription for FF/VI (all 100/25 formulation), representing less than $0.3 \%$ of the patients in the combined asthma and other diagnosis groups.

The upper-bound estimates of potential offlabel prescribing of FF/VI 200/25 that assumed all COPD patients with an asthma history received FF/VI 200/25 off-label for treatment of COPD were $16.9 \%(448 / 2653)$ when considering only the index prescription and $20.2 \%$ (535/ 2653) when considering the 87 patients who escalated from FF/VI 100/25 to FF/VI 200/25 during the 12-month study period. The lowerbound estimates of potential off-label prescribing (which assumed all COPD patients with an asthma history received FF/VI 200/25 on-label for treatment of asthma) were $7.5 \%(198 / 2653)$ at the index date and $9.3 \%(246 / 2653)$ during the 12-month follow-up period.

\section{DISCUSSION}

Findings from this retrospective, longitudinal, observational study suggest that in the years immediately following the availability of FF/VI in the UK, most FF/VI initiators (77\%) did so at the lower strength $(100 / 25)$, and of these, the majority had a recorded diagnosis of COPD. The treatment pattern data for $\mathrm{FF} / \mathrm{VI}$ indicated that most users did not have a change in FF/VI strength over a 12-month period, though this varied by diagnosis group and strength initiated.

Characteristics of new users of FF/VI 100/25, FF/VI 200/25, and other ICS/LABA FDC were largely similar within each diagnosis group in terms of demographics. Within the COPD diagnosis group, there was evidence of channelling of FF/VI to COPD patients with higher clinical severity, whereby new users of FF/VI, 
especially FF/VI 200/25, had a greater exacerbation burden and higher dyspnoea scores compared with patients who were prescribed another ICS/LABA FDC. Yet, FF/VI prescriptions were also recorded for patients with no exacerbation history recorded in primary care in the previous 12 months across the FF/VI 100/25, FF/ VI 200/25, and other ICS/LABA FDC groups $(36.5,39.3$, and $29.6 \%$ of patients, respectively, although it should be noted that the algorithm used to identify COPD exacerbations is only moderately sensitive, so these figures may overestimate the proportion of patients without an exacerbation in the prior 12 months [14]). Taken together, these observations demonstrate that ICS/LABA combinations are often correctly prescribed to patients at risk of COPD exacerbations in UK primary care [15], but may also be inappropriately prescribed to patients who do not experience exacerbations [16]. Of note, the patients experiencing exacerbations in this study who were prescribed FF/VI may also have derived more benefit from triple therapy than their prescribed combination per existing clinical evidence $[17,18]$. These observations highlight a potential disconnect between guideline recommendations for COPD maintenance and true prescribing behaviours in UK primary care. Previous studies have also demonstrated similar issues in this setting, largely regarding the overprescription of ICS/LAMA/LABA triple therapy [19]. Inappropriate ICS prescription as described here and in previous studies exposes patients to unnecessary safety issues like pneumonia [20], and also increases the healthcare resource use associated with treatment [21].

FF/VI was very rarely prescribed to children aged $<12$ years in the UK, which is consistent with the label. Few studies in the published literature have specifically examined ICS/LABA prescribing in children with asthma. In a Swedish register study, $15 \%$ of asthma medication prescriptions in children aged 0-17 were considered to be off-label [22]. In a study of prescribing in Europe, off-label prescribing of salbutamol in Dutch children $<18$ months of age was 22.7 prescriptions per 100 person years, while off-label prescribing of fixed combinations of $\beta_{2}$ mimetics plus anticholinergics in Dutch children $>6$ years of age ranged from 0.5 to 3.5 prescriptions per 100 patient-years [23]. Whilst there are no studies to directly compare $\mathrm{FF} / \mathrm{VI}$ potential off-label prescribing in children with other ICS/LABAs that also are not indicated in paediatric populations (Seretide 50/100 [salmeterol/fluticasone propionate] is licensed for children with asthma aged $\geq 4$ years in the $\mathrm{UK})$, the low level of off-label prescribing of FF/ VI in children under 12 observed suggests that physicians are aware that this new medicine is not licensed for paediatric prescribing.

Based on the upper estimate, as many as one in five new users of FF/VI with COPD in the UK were prescribed FF/VI 200/25, potentially offlabel. Defining COPD off-label prescribing in this retrospective database study was challenging because a large proportion of the COPD patients met the definition of having an asthma history, and the specific disease indication associated with each prescription of $\mathrm{FF} / \mathrm{VI}$ is not routinely recorded in electronic medical records. To overcome this challenge, we chose to present a range of off-label prescribing estimates representing the extremes of two scenarios where either all or none of the COPD patients with an asthma history were prescribed FF/VI 200/25 for their asthma. Another alternative approach would have been to estimate possible off-label prescribing only in the subgroup of COPD patients without any evidence of historical asthma. Indeed, this approach results in off-label estimates of $14.1 \%$ on the index date and $17.5 \%$ at any time during follow-up, which are within the range described when using the full COPD population.

In exploring these one in five potentially offlabel patients in more detail, we observed that many had received a high-dose ICS in the past, and over half were using a LAMA concomitantly (triple therapy), which is recommended for more severe patients [15]. In addition, these COPD patients also experienced a greater exacerbation burden. These observations may suggest that some clinicians attempt to control more severe COPD with a high dose of steroid; however, it is not possible to determine physicians' motivation from electronic medical records alone. We also noted that not all COPD patients who initiated FF/VI 200/25 continued to receive prescriptions of FF/VI 200/25 for the 
duration of follow-up; just over a quarter received only one FF/VI 200/25 prescription during follow-up, and another $26 \%$ were eventually changed to the lower FF/VI strength. In comparison, only a small proportion of $\mathrm{FF} / \mathrm{VI}$ $100 / 25$ initiators with COPD shifted to the higher strength during the 12-month study period. Our data also indicated that patients' prescriptions covered a median of 7 (FF/VI 100/25) and 5 (FF/VI 200/25) months of the study follow-up period. While it is possible that some physicians may have prescribed ICS/LABA only for acute episodes and not for maintenance during this study, we did not assess prescribing in relation to the timing of exacerbations. Alternative explanations for the low median prescription coverage may include: treatment switching and discontinuation, as noted above; loss to follow-up, as approximately a quarter of patients did not contribute a full 12 months of follow-up data, commonly as a result of switching GP practice during this period; prescribing as a therapeutic trial, whereby physicians were cautious in providing lengthy prescriptions for a novel treatment and low adherence. Among patients for whom the full 12 months of follow-up data were available, however, $44 \%$ had a medication possession ratio of at least 0.8 , and $59 \%$ had a proportion of days covered by their prescriptions of at least 0.8 , indicating a relatively high level of adherence to the prescribed treatment. Comparison studies of off-label medication use in COPD are not yet available. Within this study, it was not possible to directly compare the potential offlabel prescribing of FF/VI with the other ICS/ LABA FDC cohort; the other ICS/LABA group comprised products with varying doses of ICS, indications, and age groups, making the calculation of class level off-label prescribing inappropriate.

A strength of the study was the use of CPRD data containing rich and detailed longitudinal clinical records for a large population-based sample of patients treated for asthma and COPD in UK primary care. CPRD data have been widely used for observational research, including drug utilisation studies of prescription medications in respiratory diseases [24], and validation studies of physician-recorded diagnosis of COPD [11] and asthma [12]. While broadly representative of the UK population in terms of age, sex, and ethnicity [10], CPRD data is not a random sample. Nevertheless, the findings are likely to be applicable to other new users of FF/VI in the UK, though they may not reflect patterns in other countries.

There are several limitations inherent with the study design that should be considered when interpreting the findings. Firstly, GPs in the UK are not required to record the indication for every prescription they issue, and thus in this study we were unable to state with absolute certainty why a GP prescribed FF/VI or another ICS/LABA FDC to their patients. Secondly, there is the potential for misclassification of patients into the diagnosis groups. For example, the other diagnosis group could have included patients with COPD that did not meet the age requirement, or patients with COPD and/or asthma without a coded diagnosis in their available medical record (or only available in free text). These un- (or under)recorded COPD or asthma patients could have affected the quantification of potential off-label use. As less than $4 \%$ of new users of FF/VI had neither a COPD or asthma diagnosis, we expect any impact on the results to be minimal. We attempted to limit misclassification by using published validated diagnosis codes for asthma [12] and COPD [11]. This classification could have potentially been improved if spirometry criteria were included in the COPD diagnosis group definition; however, we observed that $73-80 \%$ of patients in the COPD group did have an $\mathrm{FEV}_{1} / \mathrm{FVC}$ ratio $<70 \%$, suggesting airflow limitation in line with a COPD diagnosis.

\section{CONCLUSION}

This study suggests that the prescribing of FF/VI is rare in children under 12 years of age in the UK. Among adults, up to one in five COPD patients in the UK have been prescribed the higher strength FF/VI 200/25 formulation. Some of this potential off-label prescribing may be linked to historical or concurrent asthma, and/or channelled to patients with more severe COPD and prior treatment with high-dose 
steroids. While the demographic characteristics of patients newly initiating FF/VI and other ICS/ LABA FDC were quite similar, there was some evidence of the channelling of FF/VI to more severe COPD patients.

\section{ACKNOWLEDGEMENTS}

Funding. CPRD conducted this study, which was funded by GlaxoSmithKline plc (study 205052). The CPRD researchers had full access to all the data used in the study, and GlaxoSmithKline plc staff had access to aggregated data. All authors take responsibility for the integrity of the data and accuracy of the data analysis. GlaxoSmithKline plc also funded article processing charges for this publication.

Medical Writing and/or Editorial Assistance. Medical writing support in the form of development of the draft outline and manuscript first draft in consultation with the authors, editorial suggestions regarding draft versions of this paper, assembling tables and figures, collating author comments, copyediting, referencing, and graphic services was provided by Catherine Amey, BSc, of GardinerCaldwell Communications, Macclesfield, UK, and was funded by GlaxoSmithKline plc.

Authorship. All named authors meet the International Committee of Medical Journal Editors criteria for authorship for this article, take responsibility for the integrity of the work as a whole, and have given their approval for this version to be published.

Authors' Contributions. Study design: DD, REG, WM, SHL. Data collection: DD, SJC, REG. Database search: SJC. Data analysis/interpretation: DD, SJC, REG, WM, CC, CH, JA, SHL. Writing/reviewing of the manuscript: DD, SJC, REG, WM, CC, CH, JA, SHL. Final approval of the manuscript: DD, SJC, REG, WM, CC, CH, JA, SHL. Guarantor: DD.

Disclosures. Trademarks are the property of their respective owners. Daniel Dedman is an employee of CPRD, which received funding from GlaxoSmithKline plc to conduct the study. Sonia J. Coton is an employee of CPRD, which received funding from GlaxoSmithKline plc to conduct the study. Rebecca E. Ghosh is an employee of CPRD, which received funding from GlaxoSmithKline plc to conduct the study. Wilhelmine Meeraus is a GSK employee and holds GSK stocks/shares. Courtney Crim is a GSK employee and holds GSK stocks/shares. Catherine Harvey is a GSK employee and holds GSK stocks/shares. Justyna Amelio is a GSK employee and holds GSK stocks/shares. Sarah H. Landis is a GSK employee and holds GSK stocks/ shares.

Compliance with Ethics Guidelines. The CPRD contains only de-identified patient data. The CPRD has broad Health Research Authority National Research Ethics Service Committee ethics and governance approval for purely observational research using the primary care data and established data linkages. The study protocol (16_229R) was approved by the CPRD Independent Scientific Advisory Committee. Tables were adapted to suppress patient counts of less than five, in compliance with CPRD policy on managing anonymisation and the risk of identification in observational research. As this study involved the secondary analysis of data collected during provision of routine care, individual informed consent was not required.

Data Availability. The datasets generated during and/or analyzed during the current study are available in the GlaxoSmithKline plc Clinical Trial Register repository, https://www. gsk-studyregister.com/. The raw CPRD data used in this study cannot be shared directly with others, but organisations may approach the CPRD to request access to the data subject to independent scientific review and contractual agreements.

Open Access. This article is distributed under the terms of the Creative Commons Attribution 4.0 International License (http:// creativecommons.org/licenses/by/4.0/), which permits unrestricted use, distribution, and reproduction in any medium, provided you give 
appropriate credit to the original author(s) and the source, provide a link to the Creative Commons license, and indicate if changes were made.

\section{REFERENCES}

1. Ejiofor S, Turner AM. Pharmacotherapies for COPD. Clin Med Insights Circ Respir Pulm Med. 2013;7:17-34.

2. Miller-Larsson A, Selroos O. Advances in asthma and COPD treatment: combination therapy with inhaled corticosteroids and long-acting beta 2-agonists. Curr Pharm Des. 2006;12:3261-79.

3. Ferguson GT, Anzueto A, Fei R, Emmett A, Knobil K, Kalberg C. Effect of fluticasone propionate/salmeterol $(250 / 50 \mathrm{mcg})$ or salmeterol $(50 \mathrm{mcg})$ on COPD exacerbations. Respir Med. 2008;102:1099-108.

4. Calverley PM, Anderson JA, Celli B, et al. Salmeterol and fluticasone propionate and survival in chronic obstructive pulmonary disease. $\mathrm{N}$ Engl J Med. 2007;356:775-89.

5. Kardos P, Wencker M, Glaab $T$, Vogelmeier C. Impact of salmeterol/fluticasone propionate versus salmeterol on exacerbations in severe chronic obstructive pulmonary disease. Am J Respir Crit Care Med. 2007;175:144-9.

6. Sharafkhaneh A, Southard JG, Goldman M, Uryniak T, Martin UJ. Effect of budesonide/formoterol pMDI on COPD exacerbations: a double-blind, randomized study. Respir Med. 2012;106:257-68.

7. Martinez FJ, Vestbo J, Anderson JA. Effect of fluticasone furoate and vilanterol on exacerbations of chronic obstructive pulmonary disease in patients with moderate airflow obstruction. Am J Respir Crit Care Med. 2017;195:881-8.

8. Ducharme FM, Ni Chroinin M, Greenstone I, Lasseson TJ. Addition of long-acting beta2-agonists to inhaled steroids versus higher dose inhaled steroids in adults and children with persistent asthma. Cochrane Database Syst Rev. 2010;14:CD005533.

9. European Medicines Agency. Assessment report for Relvar Ellipta. 2018. http://www.ema.europa.eu/ docs/en_GB/document_library/EPAR_-_Summary_ for_the_public/human/002673/WC500157636.pdf. Accessed Apr 2018.

10. Herrett E, Gallagher AM, Bhaskaran K, et al. Data resource profile: Clinical Practice Research Datalink (CPRD). Int J Epidemiol. 2015;44:1-10.
11. Quint JK, Müllerova H, DiSantostefano RL, et al. Validation of chronic obstructive pulmonary disease (COPD) recording in the Clinical Practice Research Datalink (CPRD_GOLD). BMJ Open. 2014;4:e005540.

12. Nissen F, Morales DR, Mullerova $H$, Smeeth L, Douglas IJ, Quint JK. Validation of asthma recording in the Clinical Practice Research Datalink (CPRD). BMJ Open. 2017; 7:e017474.

13. Global Initiative for Asthma (GINA). Global strategy for asthma management and prevention, updated. 2017. http://www.ginasthma.org/. Accessed Dec 2018.

14. Rothnie KJ, Müllerová H, Hurst JR, et al. Validation of the recording of acute exacerbations of COPD in UK primary care electronic healthcare records. PLoS One. 2016;11:e0151357.

15. Global Initiative for Asthma (GINA). Global strategy for for the diagnosis, management and prevention of COPD, updated. 2019. https://goldcopd. org. Accessed Dec 2019.

16. Cataldo D, Derom E, Liistro G, et al. Overuse of inhaled corticosteroids in COPD: five questions for withdrawal in daily practice. Int $\mathrm{J}$ COPD. 2018;13:2089-99.

17. Lipson D, Barnhart F, Brealey N, et al. Once-daily single-inhaler triple versus dual therapy in patients with COPD. New Eng J Med. 2018;378:1671-80.

18. Lipson D, Barnacle H, Birk R, et al. FULFIL trial: once-daily triple therapy for patients with chronic obstructive pulmonary disease. Am J Respir Crit Care Med. 2017;196:438-46.

19. Brusselle G, Price D, Gruffydd-Jones K, et al. The inevitable drift to triple therapy in COPD: an analysis of prescribing pathways in the UK. Int J Chron Obstr Pulm Dis. 2015;10:2207-17.

20. Suissa S, Coulombe J, Ernst P. Discontinuation of inhaled corticosteroids in COPD and the risk reduction of pneumonia. Chest. 2015;148(5):1177-83.

21. Chalmers JD, Poole C, Webster S, et al. Assessing the healthcare resource use associated with inappropriate prescribing of inhaled corticosteroids for people with chronic obstructive pulmonary disease (COPD) in GOLD groups A or B: an observational study using the Clinical Practice Research Datalink (CPRD). Respir Res. 2018;19:63.

22. Weidinger P, Nilsson JL, Lindblad U. Medication prescribing for asthma and COPD: a register-based cross-sectional study in Swedish primary care. BMC Fam Pract. 2014;15:54. 
23. Sen EF, Verhamme KM, Neubert A. Assessment of pediatric asthma drug use in three European countries; a TEDDY study. Eur J Pediatr. 2011;170:81-92.
24. DiSantostefano RL, Sampson T, Le HV, Hinds D, Davis KJ, Bakerly ND. Risk of pneumonia with inhaled corticosteroid versus long-acting bronchodilator regimens in chronic obstructive pulmonary disease: a new-user cohort study. PLoS One. 2014;9:e97149. 